\title{
Study on internet of things architecture of substation online monitoring equipment
}

\author{
Hejun Zhang ${ }^{1}$, Dong $\mathrm{Ge}^{1}$, Ning Yang ${ }^{2}$, Pengfei $\mathrm{Jia}^{2, *}$, Lihua $\mathrm{Li}^{2}$, and Yang Yang ${ }^{2}$ \\ ${ }^{1}$ State Grid Corporation Limited, Equipment Management Department, Beijing, China \\ ${ }^{2}$ China Electric Power Research Institute, High Voltage Research, Beijing, China
}

\begin{abstract}
This paper introduces the structure of the existing on-line monitoring system, and analyzes the problems of poor compatibility and reliability of IED in different manufacturers. Then it gives two ways to transform the on-line monitoring system of substation equipment to the Internet of things, which is to retain IED and to remove IED. On this basis, this paper introduces the application scenarios of the on-line monitoring system of substation equipment connected to the Internet of things, including accurate perception of equipment status, accurate evaluation of equipment status and intelligent research and judgment of equipment status.
\end{abstract}

\section{Introduction}

The on-line monitoring device of substation equipment has been widely used in the power grid system. In recent years, it has experienced several stages of development, and the related technology is gradually mature, and the online monitoring products are gradually increasing. Due to the detailed classification of monitoring functions, each type of on-line monitoring device is equipped with a monitoring IED to realize data acquisition, analysis, processing and IEC61850 communication protocol, which leads to a variety of IED types, high failure rate, false alarm and missing alarm.

With the development of edge computing, wireless transmission and other key technologies, as well as the full deployment of ubiquitous power Internet of things, it provides favourable conditions for the wireless on-line monitoring device of substation equipment. The sensors of all kinds of monitoring devices have gradually become wireless. The sensors, communication links and data analysis can be decoupled, which provides convenient conditions for the structure simplification and cost reduction of online monitoring devices.

\section{Application of existing on-line monitoring devices}

The composition of the current substation equipment online monitoring system is shown in Figure 1, which is composed of equipment online monitoring device, equipment online monitoring IED and station end online monitoring host (CAC).

\footnotetext{
${ }^{*}$ Corresponding author: jiapengfei@epri.sgcc.com.cn
} 


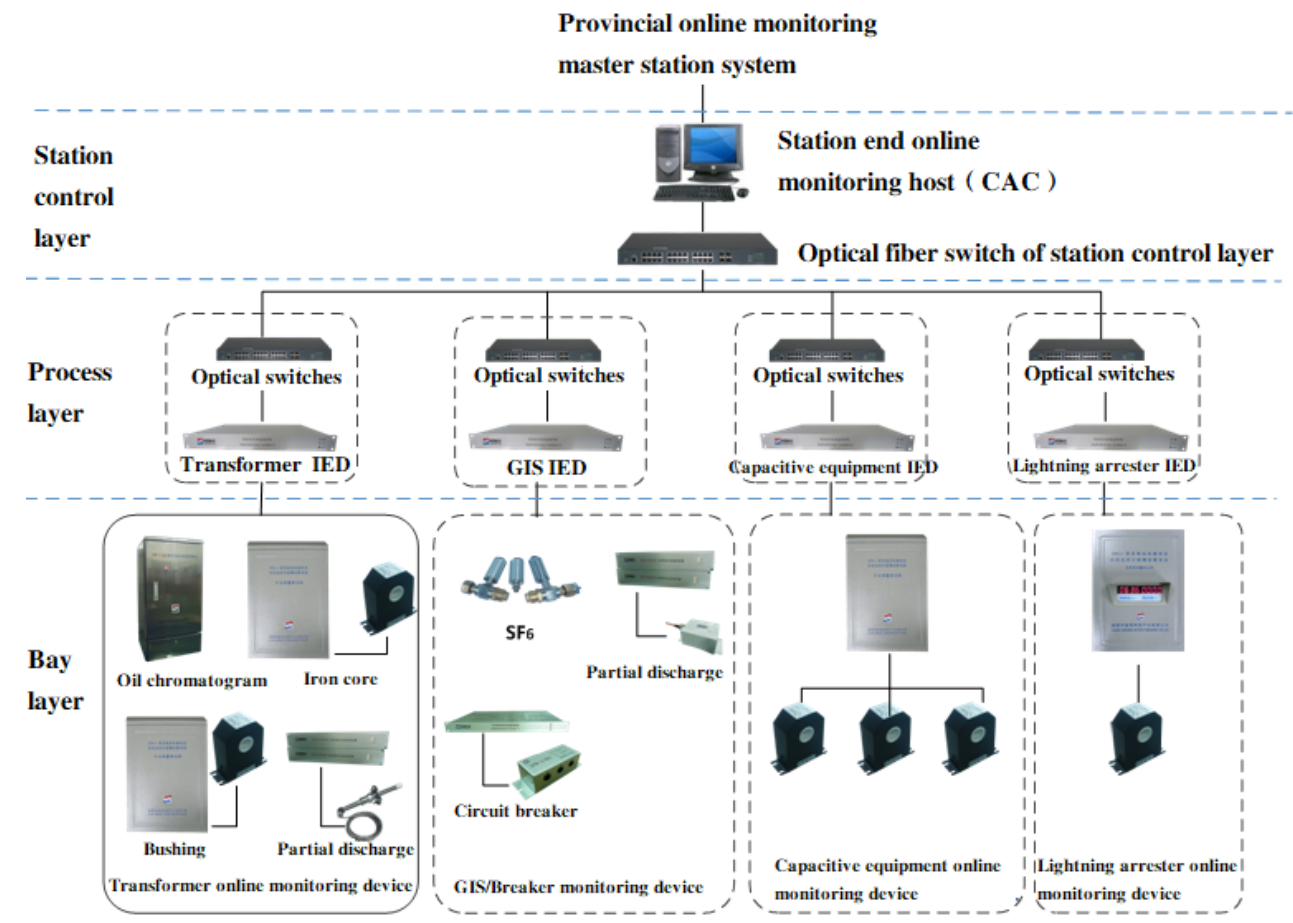

Fig. 1. On line monitoring system architecture.

(1) Equipment online monitoring device

Equipment online monitoring device refers to the monitoring device installed on or near the monitored equipment, which can automatically collect, process and send the status information of the monitored equipment, and can upload it through the field bus, Ethernet, wireless and other communication methods.

(2) Equipment online monitoring IED

Equipment on-line monitoring IED refers to the device that receives and processes the data of on-line monitoring device, and communicates with the station end online monitoring host by using the standardized protocol (IEC61850).

(3) Station end online monitoring host

The station end online monitoring host realizes the functions of on-line monitoring data analysis, display, early warning and device management, and carries out standardized communication with provincial online monitoring master station.

Currently, IED is very important in the online monitoring system. It has the function of receiving the data acquisition instructions from the monitoring center and sending the corresponding acquisition commands to each online monitoring device. After the sensors of all kinds of on-line monitoring devices complete the signal acquisition, they need to access the corresponding IED for data processing and analysis, and then embed the IED performance description file conforming to IEC61850 protocol, and then communicate with the monitoring center through optical fiber according to IEC61850 standard.

\section{Internet of things framework design}

Two ideas are considered for the wireless of online monitoring device. One is to retain the IED of the original on-line monitoring device, and change the optical fiber to wireless communication at the output end of the IED, then the sink node and the access node only 
need to transmit the IED output data. The data transmitted has met the IEC61850 protocol. Only changing the communication mode of the online monitoring device to wireless mode has the least transformation difficulty, as shown in Figure 2.

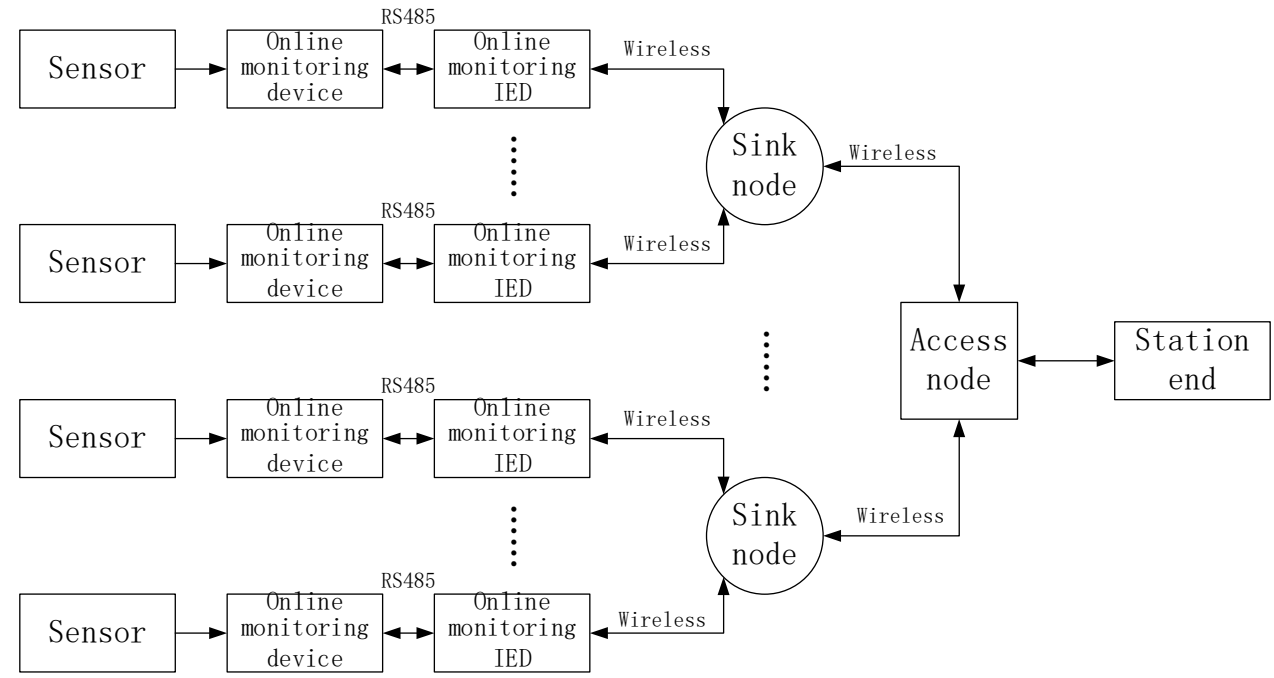

Fig. 2. IOT structure of online monitoring device with IED.

Another idea is to gradually replace IED in the Bay layer of the on-line monitoring system, as shown in Figure 3. The functions of data transmission and data analysis in IED are considered to be integrated into the sink node or on-line monitoring device, while IEC61850 communication protocol is considered to be implemented in the access node. In this way, it is necessary to establish or use a set of data communication protocols to meet the needs of wireless communication between online monitoring devices, sink nodes and access nodes. In addition, the access node needs to establish the corresponding IEC61850 model for all kinds of online monitoring, which greatly increases the complexity of access nodes.

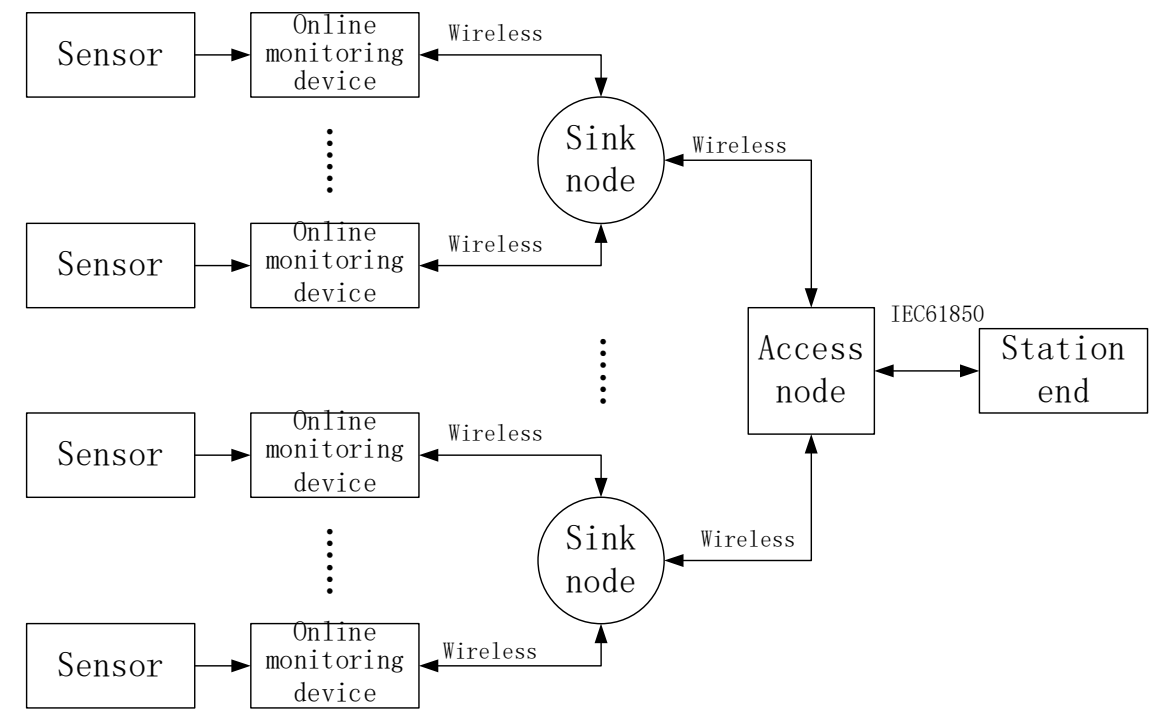

Fig. 3. IOT structure of online monitoring device without IED. 


\section{Application scenarios of device state awareness}

By collecting the monitoring information of main and auxiliary equipment, the intelligent perception of equipment status and automatic monitoring of abnormal state are realized; the intelligent evaluation system of substation equipment is improved with active fault warning as the goal; finally, a comprehensive evaluation system with equipment health status as the core is established to build a management and control platform integrating monitoring, analysis and auxiliary decision-making.

(1) Equipment status portrait

Based on the automatic collection of historical information such as account, test, transportation inspection and defects of various system equipment, a large number of advanced sensors are used to collect the environmental quantity, physical quantity, state quantity and electrical quantity of substation in real time, fully integrate the multi-source system data of operation and inspection specialty, gather multi-type equipment and environmental monitoring information, and realize the deep perception of equipment body and operation environment.

The equipment portrait module mainly includes the historical information and real-time monitoring information of equipment status. It includes the following kinds of information: basic account information, historical test information, comprehensive maintenance information, historical operation information, comprehensive defect information, live detection information, so as to realize data "one click collection"; it is convenient to access the historical detection data and other related information of the detection object and similar equipment; it combines the status evaluation information, online condition monitoring information and the production plan of the control platform Drawing information, taking substation as a unit, the key information in equipment portrait is displayed in panorama.

(2) Accurate evaluation of equipment status

It is necessary to pay attention to whether or not the general state of the equipment can be judged according to the relevant standards and regulations. When the data is abnormal, the multi state information such as live detection, bad working conditions, operation information and power failure test of the control platform is called, and other equipment information is obtained through the equipment portrait module, so as to realize the longitudinal analysis of historical data and horizontal comparative analysis of other related equipment, and conduct preliminary diagnosis on the equipment status, so as to realize the perception and early warning of equipment status. For abnormal equipment, timely push warning information to operation and maintenance personnel, adjust the state monitoring strategy, and upload it to the intelligent research and judgment module for more accurate diagnosis and analysis.

Accurate evaluation uses threshold comparison, significant difference analysis and early warning, aspect ratio analysis early warning, combined with early warning information release and query to form equipment early warning and accurate evaluation.

(3) Intelligent research and judgment of equipment status

Based on the construction of distributed storage equipment status information database and equipment defect and fault case sample database, based on the artificial intelligence learning algorithm and intelligent analysis technology such as voice recognition, image recognition, equipment status big data analysis, the association rules between equipment status and defects are established, and real-time early warning and automatic analysis of equipment defects are developed The system has the functions of active push of processing strategy and so on, forming an intelligent research and early warning mechanism of substation equipment status based on multi physical quantity perception. 


\section{Summary}

In view of the current substation operation and maintenance business, this paper plans to comprehensively use new technologies such as "big cloud and mobile intelligence" to penetrate and deeply integrate with the substation operation and maintenance business. By means of robots, equipment online monitoring, video image recognition, intelligent sensors and other means, a substation with the characteristics of "terminal ubiquitous access, platform open sharing, data-driven business, application customization" is constructed Station the integrated application system of Internet of things, improve the state control power of equipment, and promote the work of equipment inspection and on-site control to be safer, more intelligent and more efficient. It provides strong data resources support for safe and economic operation of power grid, improves business performance, and cultivates and develops strategic emerging industries, and opens up a new way for management innovation, business innovation and value creation.

We thank the State Grid Corporation of Science and Technology Project Classification Standard, Key Technology Research and Pilot Application of Substation Intelligent Equipment Based on Internet of Things Technology (grant number 5500-202055096A-0-0-00).

\section{References}

1. WANG Yi, CHEN Qixin, ZHANG Ning, et al. Fusion of the $5 \mathrm{G}$ communication and the ubiquitous electric Internet of things: Application analysis and research prospects[J]. Power System Technology, 2019, 43(5): 1575-1585.

2. WU Shanshan, NING Xin, GUO Shen, et al. Discussion on application of distribution Internet of things in new industry form[J]. High Voltage Engineering, 2019, 45(6): 1723 - 1728.

3. YANG Ting, ZHAI Feng, ZHAO Yingjie, et al. Explanation and prospect of ubiquitous electric power Internet of things[J]. Automation of Electric Power Systems, 2019, 43 (13): 9-20.

4. YANG Dongsheng, WANG Daohao, ZHOU Bowen, et al. Key technologies and application prospects of ubiquitous power internet of things[J]. Power Generation Technology, 2019, 40(2): 107-114.

5. JIANG Xiuchen, LIU Yadong, FU Xiaofei, et al. Construction ideas and development trends of transmission and distribution equipment of the ubiquitous power internet of things[J]. HighVoltageEngineering, 2019, 45(5):1345-1351. 